\title{
Constructing irreducible representations of discrete groups
}

\author{
MARC BURGER and PIERRE DE LA HARPE* \\ Institut de Mathématiques, Université de Lausanne, Dorigny, $\mathrm{CH}-1015$ Lausanne, Suisse \\ e-mail:Marc.Burger@ima.unil.ch \\ * Section de Mathématiques, Université de Genève, C.P. 240, CH-1211 Genève 24, Suisse \\ e-mail:Pierre.delaHarpe@math.unige.ch \\ MS received 11 October 1996

\begin{abstract}
The decomposition of unitary representations of a discrete group obtained by induction from a subgroup involves commensurators. In particular Mackey has shown that quasi-regular representations are irreducible if and only if the corresponding subgroups are self-commensurizing. The purpose of this work is to describe general constructions of pairs of groups $\Gamma_{0}<. \Gamma$ with $\Gamma_{0}$ its own commensurator in $\Gamma$. These constructions are then applied to groups of isometries of hyperbolic spaces and to lattices in algebraic groups.
\end{abstract}

Keywords. Commensurator subgroups; unitary representations; quasi-regular representations; Gromov hyperbolic groups; arithmetic lattices.

\section{Introduction}

Let $G$ be a separable locally compact group. The unitary dual $\hat{G}$ of $G$ is the set of equivalence classes of irreducible representations of $G$, together with its Mackey Borel structure. In this paper, "representation" means "continuous unitary representation in a separable Hilbert space".

Let us recall the definition of this structure [Dix, 18.5]. For each $n \in\{1,2, \ldots, \infty\}$, let $\operatorname{Irr}_{n}(G)$ denote the space of all irreducible representations of $G$ in a given Hilbert space of dimension $n$. The set $\operatorname{Irr}_{n}(G)$ is endowed with the topology of the weak simple convergence on $G$ (making the functions $\pi \mapsto\langle\pi(g) \xi \mid \eta\rangle$ continuous for all $g \in G$ and $\xi, \eta$ in the Hilbert space of dimension $n$ ), and with the corresponding Borel structure. The dual $\hat{G}$ is the quotient of $\bigsqcup_{1 \leq n \leq \infty} \operatorname{Irr}_{n}(G)$ by unitary equivalence, and the Mackey Borel structure on $\hat{G}$ is the quotient of the previously defined Borel structure.

In case of a countable group $\Gamma$, it follows from results of Glimm and Thoma that $\hat{\Gamma}$ is a standard Borel space if and only if $\Gamma$ is virtually abelian (see [Dix], numbers 9.1, 9.5.6 and 13.11 .12 , or [Ped, 6.8.7]); in this case the representation theory of $\Gamma$ is well understood. In all other cases there is no natural Borel coding of $\hat{\Gamma}$, i.e. $\hat{\Gamma}$ is not countably separated; for lack of a systematic procedure of constructing all irreducible representations of $\Gamma$, a natural problem is to construct large classes of irreducible representations.

Recall that two subgroups $G_{0}$ and $G_{1}$ of a group $G$ are commensurable if $G_{0} \cap G_{1}$ is of finite index in both $G_{0}$ and $G_{1}$. The commensurator of $G_{0}$ in $G$ is defined to be

$$
\operatorname{Com}_{G}\left(G_{0}\right)=\left\{g \in G \mid G_{0} \text { and } g G_{0} g^{-1} \text { are commensurable }\right\} \text {. }
$$

Let $\left(\Gamma_{i}\right)_{t \in I}$ be a family of pairwise non conjugate subgroups of a countable group $\Gamma$ such

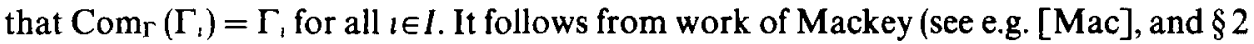


below) that unitary induction provides a well defined and injective map

$$
\bigsqcup_{i \in I} \widehat{\Gamma_{i}^{p d}} \hookrightarrow \hat{\Gamma}
$$

where $\widehat{\Gamma_{t}^{f d}}$ denotes the subset of $\hat{\Gamma}_{t}$ consisting of finite dimensional representations.

Our aim in this paper is to construct actions with noncommensurable stabilizers and pairs of groups $\Gamma_{0}<\Gamma$ such that $\operatorname{Com}_{\Gamma}\left(\Gamma_{0}\right)=\Gamma_{0}$. More generally, we construct also pairs $\Gamma_{0}<\Gamma$ such that $\Gamma_{0}$ is a subgroup of finite index in $\operatorname{Com}_{\Gamma}\left(\Gamma_{0}\right)$; in this case, the quasiregular representation of $\Gamma$ in $l^{2}\left(\Gamma / \Gamma_{0}\right)$ is a finite direct sum of irreducible representations.

In $\S 2$, we recall some classical results on unitary representations. Section 3 provides elementary examples of pairs of groups $\Gamma_{0}<\Gamma$ with $\Gamma_{0}$ its own commensurator in $\Gamma$. We consider groups of isometries of Gromov hyperbolic spaces in $\S 4$. Then, for a lattice $\Gamma$ in the group of real points of a linear algebraic group $\mathbb{G}$ defined over $\mathbb{R}$, we consider actions of $\Gamma$ on appropriate sets of maximal tori in $\S 5$ and on other sets of subgroups of $\mathbb{G}$ in $\S 6$; in each case, we find classes of irreducible quasi-regular representations of $\Gamma$.

Note on terminology. Commensurators have been known under various names, such as quasinormalizers [Cor], commensurizers [ $\mathrm{KrR}$ ] and commensurability subgroups [Mar]. We follow the terminology of [Shi, Chapter 3] and [A'B].

\section{Commensurators and induced representations}

Let $\Gamma$ be a discrete group, $\Gamma_{0}<\Gamma$ a subgroup and $\lambda_{\Gamma / \Gamma_{0}}$ the left regular representation of $\Gamma$ in $l^{2}\left(\Gamma / \Gamma_{0}\right)$.

A double class $\dot{x} \in \Gamma_{0} \backslash \operatorname{Com}_{\Gamma}\left(\Gamma_{0}\right) / \Gamma_{0}$ represented by some $x \in \operatorname{Com}_{\Gamma}\left(\Gamma_{0}\right)$ corresponds to a finite $\Gamma_{0}$-orbit $\Gamma_{0} x \Gamma_{0}$ in $\Gamma / \Gamma_{0}$, and the mapping $\Gamma_{0} \rightarrow \Gamma / \Gamma_{0}$ applying $z$ to $z x \Gamma_{0}$ induces a bijection of $\Gamma_{0} /\left(\Gamma_{0} \cap x \Gamma_{0} x^{-1}\right)$ onto $\Gamma_{0} x \Gamma_{0}$. Consequently, $\dot{x}$ gives rise to a bounded intertwining operator $T_{\dot{x}}$ of $\lambda_{\Gamma / \Gamma_{0}}$, which is defined by

$$
\left(T_{\dot{x}} f\right)\left(y \Gamma_{0}\right)=\sum_{\zeta \in \Gamma_{0} /\left(\Gamma_{0} \cap x \Gamma_{0} x^{-1}\right)} f\left(y \zeta x \Gamma_{0}\right)
$$

for all $f \in l^{2}\left(\Gamma / \Gamma_{0}\right)$ and for all $y \Gamma_{0} \in \Gamma / \Gamma_{0}$.

It is then a fact (see [Bin], Theorem 2.2) that the linear space generated by

$$
\left\{T_{\dot{x}}: l^{2}\left(\Gamma / \Gamma_{0}\right) \rightarrow l^{2}\left(\Gamma / \Gamma_{0}\right) \mid \dot{x} \in \Gamma_{0} \backslash \operatorname{Com}_{\Gamma}\left(\Gamma_{0}\right) / \Gamma_{0}\right\}
$$

is weakly dense in the space $\operatorname{Int}\left(\lambda_{\Gamma / \Gamma_{0}}\right)$ of bounded intertwining operators of $\lambda_{\Gamma / \Gamma_{0}}$. Hence, if $\Gamma_{0} \backslash \operatorname{Com}_{\Gamma}\left(\Gamma_{0}\right)$ is finite, we have

$$
\operatorname{dim} \operatorname{Int}\left(\lambda_{\Gamma / \mathrm{r}_{0}}\right)=\operatorname{Card}\left(\Gamma_{0} \backslash \operatorname{Com}_{\Gamma}\left(\Gamma_{0}\right) / \Gamma_{0}\right)
$$

and $\lambda_{\Gamma / \Gamma_{0}}$ is a finite direct sum of irreducible representations. In particular $\lambda_{\Gamma / \Gamma_{0}}$ is irreducible if and only if $\operatorname{Com}_{\Gamma}\left(\Gamma_{0}\right)=\Gamma_{0}$.

The above considerations then lead to the following theorem. Here and in the sequel we call two subgroups $\Gamma_{0}, \Gamma_{1}$ of $\Gamma$ quasiconjugate if there exists $\gamma \in \Gamma$ such that $\Gamma_{0}$ and $\gamma \Gamma_{1} \gamma^{-1}$ are commensurable.

Theorem 2.1 [Mackey]. Let $\Gamma$ be a discrete group and let $\Gamma_{0}, \Gamma_{1}$ be subgroups of $\Gamma$. (1) The representation $\lambda_{\Gamma / \Gamma_{0}}$ is irreducible if and only if $\operatorname{Com}_{\Gamma}\left(\Gamma_{0}\right)=\Gamma_{0}$, in which case $\operatorname{Ind}_{\Gamma_{0}}^{\Gamma}(\pi)$ is irreducible for any $\pi \in \Gamma_{0}^{\rho^{d d}}$, and unitary induction 


$$
\operatorname{Ind}_{\Gamma_{0}}^{\Gamma}: \widehat{\Gamma_{0}^{f d}} \rightarrow \hat{\Gamma}
$$

is an injective map.

(2) If $\operatorname{Com}_{\Gamma}\left(\Gamma_{i}\right)=\Gamma_{i}, i=0,1$, then $\lambda_{\Gamma / \Gamma_{0}}$ and $\lambda_{\Gamma / \Gamma_{1}}$ are unitarily equivalent if and only if $\Gamma_{0}$ and $\Gamma_{1}$ are quasiconjugate in $\Gamma$.

In case $\Gamma_{0}$ and $\Gamma_{1}$ are not quasiconjugate in $\Gamma$, if $\pi_{0}$, respectively $\pi_{1}$, are finite dimensional irreducible unitary representations of $\Gamma_{0}$, respectively $\Gamma_{1}$, then $\operatorname{Ind}_{\Gamma_{0}}^{\Gamma}\left(\pi_{0}\right)$ and $\operatorname{Ind}_{\Gamma_{1}}^{\Gamma}\left(\pi_{1}\right)$ are not equivalent.

Remark. We do not know whether the condition $\pi \in \widehat{\Gamma_{0}^{f d}}$ in (1) can be replaced by $\pi \in \widehat{\Gamma}_{0}$.

Let us restate the previous Theorem in a slightly different way. Let $\Gamma$ be a discrete group acting on a set $A$, and denote by

$$
\mathscr{Z}_{\Gamma}(a) \doteqdot\{\gamma \in \Gamma \mid \gamma a=a\}
$$

the stabilizer of a point $a \in A$; if more precision is needed, we write $\mathscr{Z}_{\Gamma, A}(a)$ for $\mathscr{Z}_{\Gamma}(a)$.

\section{DEFINITION}

The action $\mathrm{\Gamma} \times A \longrightarrow A$ has noncommensurable stabilizers (N.C.S.) if any two points $a_{1}$, $a_{2} \in A$ with commensurable stabilizers coincide.

The following lemma is an easy observation.

Lemma 2.2. (1) Let $\Gamma \times A \longrightarrow A$ be a N.C.S. action. For $a_{1}, a_{2} \in A$ and $\gamma \in \Gamma$, we have $\gamma a_{1}=a_{2}$ if and only if $\gamma \mathscr{Z}_{\Gamma}\left(a_{1}\right) \gamma^{-1}=\mathscr{Z}_{\Gamma}\left(a_{2}\right)$, if and only if $\gamma \mathscr{Z}_{\Gamma}\left(a_{1}\right) \gamma^{-1}$ and $\mathscr{Z}_{\Gamma}\left(a_{2}\right)$ are commensurable.

In particular $\left(\mathscr{Z}_{\Gamma}(a)\right)_{a \in A}$ is a set of self-commensurizing subgroups of $\Gamma$, two subgroups $Z_{\Gamma}\left(a_{1}\right), Z_{\Gamma}\left(a_{2}\right)$ of the set being quasiconjugate if and only if $a_{1}, a_{2}$ are in the same $\Gamma$-orbit. (2) Let $\mathscr{G}$ be a set of self-commensurizing subgroups of $\Gamma$ which is stable under conjugation. Then the action of $\Gamma$ on $\mathscr{G}$ by conjugation is N.C.S.

It follows from Theorem 2.1 and Lemma 2.2. that, for a N.C.S. action $\Gamma \times A \rightarrow A$, unitary induction

$$
\text { Ind: } \bigsqcup_{a \in \Gamma \backslash A} \overline{\mathscr{Z}_{\Gamma}(a)^{f d} \longrightarrow \hat{\Gamma}}
$$

is an injective map.

For later use we record the following general fact. Let $\pi, \rho$ be unitary representations of a group $\Gamma$. We write $\pi<\rho$ to express that $\pi$ is weakly contained in $\rho$ [Dix, 18.1.3], and $\pi \sim \rho$ to express that $\pi$ and $\rho$ are weakly equivalent [namely that $\pi \prec \rho$ and $\rho \prec \pi$ ].

Lemma 2.3. Let $\Gamma_{0}$ be a subgroup of $\Gamma$. Then $\lambda_{\Gamma / \Gamma_{0}} \prec \lambda_{\Gamma}$ if and only if $\Gamma_{0}$ is amenable.

Proof. If $\Gamma_{0}$ is amenable, $1_{\Gamma_{0}} \prec \lambda_{\Gamma_{0}}$ and hence $\lambda_{\Gamma / \Gamma_{0}}=\operatorname{Ind}_{\Gamma_{0}}^{\Gamma}\left(1_{\Gamma_{0}}\right) \prec \operatorname{Ind}_{\Gamma_{0}}^{\Gamma}\left(\lambda_{\Gamma_{0}}\right)=\lambda_{\Gamma}$.

Conversely, since $1_{\Gamma_{0}}$ is contained in $\operatorname{Res}_{\Gamma_{0}}\left(\lambda_{\Gamma / \Gamma_{0}}\right)$ and since $\operatorname{Res}_{\Gamma_{0}}\left(\lambda_{\Gamma}\right)$ is a multiple of $\lambda_{\Gamma_{0}}$, the assumption $\lambda_{\Gamma / \Gamma_{0}}<\lambda_{\Gamma}$ implies

$$
1_{\Gamma_{0}}<\operatorname{Res}_{\Gamma_{0}}\left(\lambda_{\Gamma / \Gamma_{0}}\right)<\operatorname{Res}_{\Gamma_{0}}\left(\lambda_{\Gamma}\right) \sim \lambda_{\Gamma_{0}}
$$

and hence $\Gamma_{0}$ is amenable. 


\section{Elementary examples of N.C.S. actions}

Define a group action $G \times A \rightarrow A$ to be large if, for all $a \in A$, all $\mathscr{Z}_{G}(a)$-orbits in $A \backslash\{a\}$ are infinite. The next lemma is a convenient tool for constructing N.C.S. actions.

Lemma 3.1. (1) A large action is N.C.S.

(2) Let $G \times A \rightarrow A$ be a large transitive action and let $\Gamma<G$ be a subgroup such that $\operatorname{Com}_{G} \Gamma=G$. Assume that there exists a point $a_{0} \in A$ such that all $\mathscr{Z}_{\Gamma, A}\left(a_{0}\right)$-orbits in $A \backslash\left\{a_{0}\right\}$ are infinite. Then the restricted action $\Gamma \times A \rightarrow A$ is large.

Proof. (1) For a large action $G \times A \rightarrow A$ and for two points $a_{1}, a_{2} \in A$ with $\mathscr{Z}_{G}\left(a_{1}\right)$ and $\mathscr{Z}_{G}\left(a_{2}\right)$ commensurable, the $\mathscr{Z}_{G}\left(a_{1}\right)$-òrbit of $a_{2}$ is finite and hence $a_{1}=a_{2}$.

(2) For $a \in A$ and $g \in G$ such that $g a_{0}=a$, the $\mathscr{Z}_{\Gamma, A}(a)$-orbits in $A \backslash\{a\}$ are infinite if and only if the $\left(g^{-1} \mathscr{Z}_{\Gamma, A}(a) g\right)$-arbits in $A \backslash\left\{a_{0}\right\}$ are infinite. Since

$$
g^{-1} \mathscr{Z}_{\Gamma, A}(a) g=g^{-1} \Gamma g \cap \mathscr{Z}_{G, A}\left(a_{0}\right)
$$

and $G=\operatorname{Com}_{G} \Gamma$, the subgroup

$$
\Delta_{0} \doteqdot \mathscr{Z}_{\Gamma, A}\left(a_{0}\right) \cap g^{-1} \mathscr{Z}_{\Gamma, A}(a) g=Z_{\Gamma, A}\left(a_{0}\right) \cap g^{-1} \Gamma g
$$

is of finite index in $\mathscr{Z}_{\Gamma, A}\left(a_{0}\right)$. In particular all $\Delta_{0}$-orbits in $A \backslash\left\{a_{0}\right\}$ are infinite and the same holds therefore for $g^{-1} Z_{\Gamma, A}(a) g$.

(Claim (1) of Lemma 3.1 is a straightforward generalization of Theorem 4 in [Oba], which delas with doubly transitive actions on infinite sets.)

Example 1. Let $\mathbb{B}$ be an infinite field and let $\mathrm{Gr}_{k}\left(\mathbb{K}^{n}\right)$ denote the Grassmannian of $k$-dimensional subspaces of $\mathbb{K}^{n}$, where $n, k$ are integers with $n \geqslant 2$ and $1 \leqslant k \leqslant n-1$.

The natural action of $G L(n, \mathbb{K})$ on $\mathrm{Gr}_{k}\left(\mathbb{K}^{n}\right)$ is N.C.S.

If $\mathbb{K}$ is a number field and if $\mathcal{O}_{K}$ denotes its ring of integers, the action of $G L\left(n, \mathcal{O}_{K}\right)$ on $\mathrm{Gr}_{k}\left(\mathbb{K}^{n}\right)$ is N.C.S.

Proof. For two distinct points $y_{1}, y_{2}$ in $\mathrm{Gr}_{k}\left(\mathbb{K}^{n}\right)$, the maximal parabolic subgroup

$$
P_{y_{1}} \doteqdot\left\{g \in G L(n, \mathbb{K}) \mid g y_{1}=y_{1}\right\}
$$

acts transitively on the infinite subset

$$
\left\{y \in \mathrm{Gr}_{k}\left(\mathbb{K}^{n}\right) \mid \operatorname{dim}_{K}\left(y \cap y_{1}\right)=\operatorname{dim}_{K}\left(y_{2} \cap y_{1}\right)\right\}
$$

of the Grassmannian. Hence the transitive action of $G L(n, \mathbb{K})$ on $\mathrm{Gr}_{k}\left(\mathbb{K}^{n}\right)$ is large; in particular $P_{y}$ is its own commensurator in $G L(n, \mathbb{K})$ for all $y \in G_{k}\left(\mathbb{K}^{n}\right)$.

Let $\mathbb{K}$ be now a number field. If $y_{0} \in \mathrm{Gr}_{k}\left(\mathbb{K}^{n}\right)$ denote the subspace spanned by the first $k$ vectors of the canonical basis of $\mathbb{K}^{n}$ and if $\Gamma=G L\left(n, \mathcal{O}_{K}\right)$, one has

$$
\mathscr{Z}_{\Gamma}\left(y_{0}\right)=\left(\gamma \in \Gamma \mid \gamma \text { of the form }\left(\begin{array}{cccccc}
* & \ldots & * & * & \ldots & * \\
\vdots & \vdots & \vdots & \vdots & \vdots & \vdots \\
* & \ldots & * & * & \ldots & * \\
0 & \ldots & 0 & * & \ldots & * \\
\vdots & \vdots & \vdots & \vdots & \vdots & \vdots \\
0 & \cdots & 0 & * & \ldots & *
\end{array}\right)\right)
$$


(with the block of zeros having $n-k$ rows and $k$ columns). Let $y_{1} \in \mathrm{Gr}_{k}\left(\mathbb{R}^{n}\right) \backslash\left\{y_{0}\right\}$; set $l=k-\operatorname{dim}_{\mathbb{K}}\left(y_{0} \cap y_{1}\right)$. We identify $\mathbb{K}^{n} / y_{0}$ with the vector space $\mathbb{K}^{n-k}$. The actions of $P_{y_{0}}$ on $\mathbb{K}^{n}$ and on $\left\{g \in \mathrm{Gr}_{k}\left(\mathbb{K}^{n}\right) \mid \operatorname{dim}\left(y \cap y_{0}\right)=\operatorname{dim}\left(y_{1} \cap y_{0}\right)\right\}$ factor as actions of $G L(n-k, \mathbb{K})$ on $\mathbb{K}^{n-k}$ and $\mathrm{Gr}_{l}\left(\mathbb{K}^{n-k}\right)$ respectively, so that the action of $\mathscr{Z}_{\Gamma}\left(y_{0}\right)$ on $\mathrm{Gr}_{k}\left(\mathbb{K}^{n}\right) \backslash\left\{y_{0}\right\}$ factors as an action of $G L\left(n-k, \mathcal{O}_{k}\right)$ on $\mathrm{Gr}_{l}\left(\mathbb{K}^{n-k}\right)$. The latter action has clearly all its orbits infinite, since the Zariski closure of $G L\left(n-k, \mathcal{O}_{K}\right)$ contains that of $G L(n-k, \mathbb{Z})$ and thus contains $S L(n-k, \mathbb{C})$. It follows first that all orbits of $\mathscr{Z}_{\Gamma}\left(y_{0}\right)$ on $\mathrm{Gr}_{k}\left(\mathbb{K}^{n}\right) \backslash\left\{y_{0}\right\}$ are infinite, and second that $\mathscr{Z}_{\Gamma}(y)=\Gamma \cap P_{y}$ is its own commensurator in $\Gamma=G L\left(n, \mathcal{O}_{k}\right)$ for all $y \in \mathrm{Gr}_{k}\left(\mathbb{K}^{n}\right)$.

We observe the following consequence of Example 1.

\section{PROPOSITION 3.2}

The unitary representation $\pi$ of $S L(n, \mathbb{Z})$ in $L^{2}\left(\mathbb{R}^{n} / \mathbb{Z}^{n}\right)$ is an orthogonal direct sum of irreducible representations.

Proof. By Fourier transform, $\pi$ is equivalent to the permutation representation of $S L(n, \mathbb{Z})$ in $l^{2}\left(\mathbb{Z}^{n}\right)$; the latter is a direct sum of quasi-regular representations $\pi_{k} \doteqdot \lambda_{S L(n, \mathbb{Z}) / \Gamma_{k}}$, where $\Gamma_{k}$ denotes the stabilizer of $(k, 0, \ldots, 0) \in \mathbb{Z}^{n}$ in $S L(n, \mathbb{Z})$, for all $k \geqslant 0$. The one-dimensional representation $\pi_{0}$ is irreducible. For $k \geqslant 1$, and $\Gamma_{k}^{\prime}$ the stabilizer of $(k: 0: \cdots: 0) \in \mathbb{P}^{n-1}(\mathbb{Q})$, Mackey's result and Example 1 imply that $\lambda_{S L(n, \mathbb{Z}) / \Gamma_{k}^{\prime}}$ is irreducible. As $\Gamma_{k}$ is of index 2 in $\Gamma_{k}^{\prime}$, the representation $\pi_{k}$ is either irreducible or sum of 2 irreducibles.

For a group action $G \times A \rightarrow A$ and subsets $B \subset A, S \subset G$ we set

$$
\begin{aligned}
& \mathscr{Z}_{G, A}(B) \doteqdot \bigcap_{b \in B} \mathscr{Z}_{G, A}(b) \\
& \mathscr{N}_{G, A}(B) \doteqdot\{g \in G \mid g(B)=B\}
\end{aligned}
$$

and $\mathscr{F}_{A}(S)$ the set of common fixed points of elements in $S$. Observe that

$$
\mathscr{N}_{G, A}(B)=\mathscr{Z}_{G, \mathcal{P}(A)}(B)
$$

where $\mathscr{P}(A)$ denotes the power set of $A$.

Lemma 3.3. Let $G \times A \rightarrow A$ be an action and let $S \subset G$ be a union of conjugacy classes of $G$ such that

$$
\mathscr{F}_{A}(g)=\mathscr{F}_{A}\left(g^{n}\right) \text { and }\left|\mathscr{F}_{A}(g)\right|<\infty
$$

for all $g \in S$ and for all $n>1$. Then the action of $G$ on the set

$$
\left\{F \in \mathscr{P}(A) \mid F=\mathscr{F}_{A}(g) \text { for some } g \in S\right\}
$$

is N.C.S.

Proof. Let $g, h \in S$ be such that the subgroups $\mathscr{N}_{G, A}\left(\mathscr{F}_{A}(g)\right)$ and $\mathscr{N}_{G, A}\left(\mathscr{F}_{A}(h)\right)$ are commensurable in $G$. Since $\mathscr{F}_{A}(g)$ and $\mathscr{F}_{A}(h)$ are both finite subsets of $A$, the subgroup

$$
K \doteqdot \mathscr{Z}_{G, A}\left(\mathscr{F}_{A}(g)\right) \cap \mathscr{X}_{G, A}\left(\mathscr{F}_{A}(h)\right)
$$

is of finite index in $\mathscr{Z}_{G, A}\left(\mathscr{F}_{A}(g)\right)$ and $\mathscr{Z}_{G, A}\left(\mathscr{F}_{A}(h)\right)$. 
Hence there exists an integer $N \geqslant 1$ such that $g^{N}$ and $h^{N}$ are in $K$. One has

$$
\mathscr{F}_{A}(g)=\mathscr{F}_{A}\left(g^{N}\right) \supset \mathscr{F}_{A}(K) \supset \mathscr{F}_{A}\left(\mathscr{Z}_{G, A}\left(\mathscr{F}_{A}(h)\right)\right)=\mathscr{F}_{A}(h)
$$

and similarly $\mathscr{F}_{A}(h) \supset \mathscr{F}_{A}(g)$, so that $\mathscr{F}_{A}(h)=\mathscr{F}_{A}(g)$.

Example 2. Consider a subgroup $\Gamma$ of $S L(n, \mathbb{C})$ and an element $\gamma \in \Gamma$ which is diagonalizable with eigenvalues $\lambda_{1}, \ldots, \lambda_{n}$ and which is regular in the following sense: one has $\lambda_{j}^{N} \neq \lambda_{k}^{N}$ for each integer $N \geqslant 1$ whenever $j, k$ are distinct in $\{1, \ldots, n\}$; in other words, the fixed point set $\mathscr{F}(\gamma)$ of $\gamma$ in $\mathbb{P}^{n-1}(\mathbb{C})$ has cardinality $n$ and $\mathscr{F}\left(\gamma^{N}\right)=\mathscr{F}(\gamma)$ for all integers $N \in \mathbb{Z}, N \neq 0$. Then the subgroup

$$
\mathscr{N}_{\Gamma, \mathbb{P}^{n-1}(\mathcal{C})}(\mathscr{F}(\gamma))=\left\{\gamma^{\prime} \in \Gamma \mid \gamma^{\prime} \text { permutes the eigen-directions of } \gamma\right\}
$$

of $\Gamma$ is its own commensurator in $\Gamma$ by Lemma 3.3. (This subgroup of $\Gamma$ is distinct from $\Gamma$ itself as soon as $\Gamma$ is not virtually abelian.)

Observe that the group

$$
T \doteqdot \mathscr{X}_{S L(n, \mathrm{C}), \mathrm{P}^{*-1}(\mathrm{C})}(\mathscr{F}(\gamma))
$$

is a maximal torus in $S L(n, \mathbb{C})$ and that $\mathscr{N}_{\Gamma, \mathbf{p}^{n-1}(\mathcal{C})}(\mathscr{F}(\gamma))$ is the intersection with $\Gamma$ of the normalizer of $\mathbb{T}$ in $S L(n, \mathbb{C})$. More on this in $\S 5$ below.

Example 3. Consider an integer $n \geq 2$, the group $\Gamma=S L(n, \mathbb{Z})$ and the subgroup $\Gamma_{0}$ of upper triangular matrices in $\Gamma$ (with diagonal entries \pm 1 ).

Then $\Gamma_{0}$ is its own commensurator in $\Gamma$.

Proof. Let Flag $\left(\mathbb{C}^{n}\right)$ be the set of complete flags in $\mathbb{C}^{n}$. Let $S$ be the subset of $\Gamma$ consisting of matrices which have precisely one Jordan block. Then, for the action of $\Gamma$ on Flag $\left(\mathbb{C}^{n}\right)$, one has $\mathscr{F}(\gamma)=\mathscr{F}\left(\gamma^{n}\right)$ and $|\mathscr{F}(\gamma)|=1$ for all $\gamma \in S$. This ends the proof because $\Gamma_{0}$ is the stabilizer of the flag $\mathbb{C} \subset \mathbb{C}^{2} \subset \cdots \subset \mathbb{C}^{n-1}$ associated to the canonical basis of $\mathbb{C}^{n}$.

Consider the group $\Gamma=S L(3, \mathbb{Z})$. For a subgroup $\Gamma_{0}=\Gamma \cap P_{y}$ as in Example 1, it follows from Lemma 2.3 that the irreducible representation $\lambda_{\Gamma / \Gamma_{0}}$ is not weakly contained in $\lambda_{\Gamma}$. But for a subgroup $\Gamma_{0}=\mathscr{N}_{\Gamma, p^{n-1}(\mathbb{C})}(\mathscr{F}(\gamma))$ as in Example 2 or for the triangular subgroup $\Gamma_{0}$ of Example 3, one has $\lambda_{\Gamma / \Gamma_{0}} \prec \lambda_{T}$ by Lemma 2.3, and consequently $\lambda_{\Gamma / \Gamma_{0}} \sim \lambda_{\Gamma}$ by $[\mathrm{BCH}]$.

There are examples of self-commensurizing subgroups of braid groups and of related groups in [FRZ] and in [Par].

\section{Groups of isometries of hyperbolic spaces}

4.1. Let $X$ be a Gromov hyperbolic space; let $X(\infty)$ be its Gromov boundary and Is $(X)$ its group of isometries. Then Is $(X)$ acts on $X(\infty)$ and on $S^{2} X(\infty)$, the set of unordered pairs of points in $X(\infty)$.

Let $\Gamma$ be a subgroup of Is $(X)$. Denote by $X(\infty)_{p} \subset X(\infty)$ the set of fixed points of parabolic elements in $\Gamma$ and by $S^{2} X(\infty)_{h} \subset S^{2} X(\infty)$ the set of fixed point sets of hyperbolic elements in $\Gamma$. 


\section{PROPOSITION 4.1}

The action of $\Gamma$ on

$$
X(\infty)_{p} \bigsqcup S^{2} X(\infty)_{h}
$$

has noncommensurable stabilizers.

Proof. Let $\Gamma_{\text {ne }}$ denote the set of non elliptic elements in $\Gamma$. For the $\Gamma$-action on $X(\infty)$ and for each $\gamma \in \Gamma_{\text {ne }}$, one has

$$
\mathscr{F}_{X(\infty)}(\gamma)=\mathscr{F}_{X(\infty)}\left(\gamma^{n}\right) \text { for all } n \geq 1
$$

and $\mathscr{F}_{X(\infty)}(\gamma)$ is of cardinality 1 or 2 depending on whether $\gamma$ is parabolic or hyperbolic. Thus Proposition 4.1 follows from Lemma 3.3.

Remark. For each hyperbolic element $\gamma \in \Gamma$, recall that the cyclic group $\gamma^{\mathbb{Z}}$ is of finite index in the group $\mathscr{Z}=\mathscr{Z}_{\Gamma, S^{2} X(\infty)}\left(\mathscr{F}_{X(\infty)}(\gamma)\right)$; see e.g. [GhH, chap. 8, $\mathrm{n}^{0} 33$ ]; in particular, the group $\mathscr{Z}$ is amenable. By Lemma 2.3 , the quasi-regular representation $\lambda_{\Gamma / \mathscr{L}}$ is weakly contained in the regular representation $\lambda_{\Gamma}$.

Assume moreover that $X$ is a discrete space which has at most exponential growth and that $\Gamma \subset \operatorname{Is}(X)$ is a discrete subgroup. For each parabolic element $\gamma \in \Gamma$, the group $\mathscr{Z}=\mathscr{Z}_{\Gamma, X(\infty)}\left(\mathscr{F}_{X(\infty)}(\gamma)\right)$ is amenable (see Proposition 1.6 in [BuM]), so that one has also $\lambda_{\Gamma / \mathscr{Z}} \prec \lambda_{\Gamma}$. Indeed, the set

$$
\left\{\mathscr{Z}_{\Gamma, X(\infty)}(\omega) \mid \omega \in X(\infty)_{p} \bigsqcup S^{2} X(\infty)_{h}\right\}
$$

coincides with the set of all maximal amenable infinite subgroups of $\Gamma$ [Ada].

In case $\Gamma$ is a Gromov hyperbolic group, the set $X(\infty)_{p}$ is empty because there is no parabolic. If $\Gamma$ is moreover torsion free, then $\mathscr{Z}_{\Gamma}(\omega)$ is infinite cyclic for all $\omega \in S^{2} X(\infty)_{h}$.

It is known that the reduced $C^{*}$-algebra of a torsion free Gromov hyperbolic group $\Gamma$ is simple [Har]. From this and Lemma 2.3, it follows that the quasi-regular representation $\lambda_{\Gamma / \mathscr{R}_{\Gamma}(\omega)}$ is quasi-equivalent to the regular representation $\lambda_{\Gamma}$ for each $\omega \in S^{2} X(\infty)_{h}$.

For a nonabelian free group, this is Proposition 1 of [Boz], itself a paper strongly motivated by [Yos].

\subsection{Let now $X$ be a proper CAT(-1)-space and let}

$$
\mathscr{G} X=\{c: \mathbb{R} \rightarrow X \mid c \text { is isometric }\}
$$

be the space of parametrized geodesics in $X$ with the topology of uniform convergence on compactas. The action of $\mathbb{R}$ on $\mathscr{G} X$ via reparametrizations

$$
g_{t} c(s)=c(s+t), \quad c \in \mathscr{G} X, \quad s, t \in \mathbb{R}
$$

commutes with that of $\operatorname{Is}(X)$ and defines for any discrete subgroup $\Gamma<\operatorname{Is}(X)$ a flow on $\Gamma \backslash \mathscr{G} X$, called the geodesic flow. We recall that, for a discrete divergence group $\Gamma<\operatorname{Is}(X)$, there is a canonical Patterson-Sullivan measure $m_{\mathrm{ps}}$ on $\Gamma \backslash \mathscr{G} X$ which is invariant and ergodic for the geodesic flow. The notion of a divergence group is borrowed from Patterson-Sullivan theory of Kleinian groups ([Pat], [Sul]; see also [Bou], [Coo], [CoP] which is generalized to CAT(-1)-spaces in [BuM]). 


\section{PROPOSITION 4.2}

Let $\Lambda<\operatorname{Is}(X)$ be a discrete subgroup. Let

$$
\mathscr{S}(\Lambda)=\left\{\Gamma<\Lambda \mid \Gamma \text { is a divergence group with } m_{\mathrm{PS}}(\Gamma \backslash \mathscr{G} X)<\infty\right\}
$$

be endowed with the ordering given by inclusion and let $\mathscr{C} \subset \mathscr{I}(\Lambda)$ be a commensurability class.

Then $\mathscr{C}$ has a unique maximal element $\Gamma_{\mathscr{C}}$, and this subgroup $\Gamma_{\mathscr{G}}$ satisfies $\operatorname{Com}_{\Lambda} \Gamma_{\mathscr{6}}=\Gamma_{\mathscr{q}}$. Moreover, if $\sim$ denotes the relation of commensurability on $\mathscr{I}(\Lambda)$, the action of $\Lambda$ on $\mathscr{S}(\Lambda) / \sim$ by conjugation is $N . C . S$.

In particular, for each $\Gamma<\mathscr{S}(\Lambda)$, the quasi-regular representation $\lambda_{\Lambda / \Gamma}$ is a finite sum of irreducible representations; if $\Gamma_{+}=\operatorname{Com}_{\Lambda}(\Gamma)$, then $\Gamma$ is of finite index in $\Gamma_{+}$and $\lambda_{\Lambda / \Gamma_{+}}$is irreducible.

Remarks. (i) Let $\Gamma<$ Is $(X)$ be a non-elementary discrete subgroup, $\mathscr{L}_{\Gamma} \subset X(\infty)$ its limit set and $Q_{\Gamma}=\operatorname{Co}\left(\mathscr{L}_{\Gamma}\right) \subset X$ the convex hull of the latter. If $\Gamma \backslash Q_{\Gamma}$ is compact (that is, if $\Gamma$ is convex-cocompact) then $\Gamma$ is a divergence group with $m_{\mathrm{PS}}(\Gamma \backslash \mathscr{G} X)<\infty$; see [Bou].

(ii) Let $X$ be a symmetric space of rank 1 and $\Gamma<\operatorname{Is}(X)$ a geometrically finite subgroup (see [Bow]). Then $\Gamma$ is a divergence group with $m_{\mathrm{PS}}(\Gamma \backslash \mathscr{G} X)<\infty$.

Example. Let $\Lambda<\operatorname{PSL}(2, \mathbb{R})$ be a discrete subgroup. Then $\mathscr{P}(\Lambda)$ contains all finitely generated non virtually cyclic subgroups of $\Lambda$. Indeed, such subgroups are nonelementary and geometrically finite.

Thus, for a finitely generated infinite subgroup $\Gamma$ of $\Lambda$, the quasi-regular representation $\lambda_{\Lambda / \Gamma}$ is a finite sum of irreducible representations: this follows from Proposition 4.1 if $\Gamma$ is virtually cyclic, in which case $\lambda_{\Lambda / \Gamma} \prec \lambda_{\Lambda}$, and from Proposition 4.2 in other cases, for which $\lambda_{\Lambda / \Gamma} \nless \lambda_{\Lambda}$.

Proof of Proposition 4.2. It suffices to show that, given a discrete divergence group $\Gamma_{0}<\operatorname{Is}(X)$ with $m_{\mathrm{PS}}\left(\Gamma_{0} \backslash \mathscr{G} X\right)<\infty$ and a discrete subgroup $\Gamma<\mathrm{Is}(X)$ with $\Gamma_{0}<\Gamma<\operatorname{Com}_{\mathrm{Is}(X)}\left(\Gamma_{0}\right)$, the subgroup $\Gamma_{0}$ is of finite index in $\Gamma$.

Indeed, assuming this is true, consider the commensurability class $\mathscr{C}$ of a subgroup $\Gamma_{0}$ of $\Lambda$ which is in $\mathscr{S}(\Lambda)$. Setting $\Gamma_{\mathscr{C}}=\operatorname{Com}_{\Lambda}\left(\Gamma_{0}\right)$ one has $\Gamma_{0}$ of finite index in $\Gamma_{\mathscr{C}} ;$ one has therefore $\Gamma_{\mathscr{G}} \in \mathscr{S}(\Lambda)$ and $\operatorname{Com}_{\Lambda} \Gamma_{\mathscr{Q}}=\Gamma_{\mathscr{G}}$. As any group commensurable with $\Gamma_{0}$ is in $\Gamma_{\mathscr{Q}}$, the latter group is clearly the unique maximal element of $\mathscr{C}$. The last claim of the proposition is now obvious.

For the convenience of the reader we recall the construction of $m_{\mathrm{PS}}$ (see $\S 1.3$ in [BuM]). Let $\delta$ be the critical exponent of $\Gamma_{0}$, let $\mu: X \rightarrow M^{+}(X(\infty))$ be the $\delta$-dimensional Patterson-Sullivan density for $\Gamma_{0}$ and let $(\xi \mid \eta)_{x}$ denote the Gromov scalar product of $\xi$, $\eta \in X(\infty)$. Using the $\Gamma$-invariant measure

$$
\frac{\mathrm{d} \mu_{x}(\xi) \times \mathrm{d} \mu_{y}(\xi)}{\mathrm{e}^{-2 \delta(5) m)_{x}}}
$$

on $X(\infty) \times X(\infty) \backslash\{$ diagonal\}, one obtains a $\Gamma$-invariant and geodesic-flow invariant measure $\tilde{m}_{\mu}$ on $\mathscr{G} X$; the Patterson-Sullivan measure $m_{\mathrm{PS}}$ is then the corresponding geodesic-flow invariant measure on $\Gamma \backslash \mathscr{G} X$. 
We recall furthermore that $\gamma_{*} \mu_{x}=\mu_{y x}$ for all $\gamma \in \Gamma_{0}, x \in X$, and that there exists a homomorphism $\chi: \operatorname{Com}_{\mathrm{Is}(X)}\left(\Gamma_{0}\right) \rightarrow \mathbb{R}_{+}^{*}$ such that $\gamma_{*} \mu_{x}=\chi(\gamma) \mu_{x}$ for all $\gamma \in \operatorname{Com}_{\mathrm{Is}(X)}\left(\Gamma_{0}\right)$, $x \in X$. From this follows $\gamma_{*} \tilde{m}_{\mu}=\chi(\gamma)^{2} \tilde{m}_{\mu}$ for all $\gamma \in \operatorname{Com}_{\mathrm{Is}(X)}\left(\Gamma_{0}\right)$ (see [BuM], Corollary 6.5.3).

Since $\Gamma$ acts properly discontinuously on $\mathscr{G} X$, there exists a compact set $K \subset \mathscr{G} X$ of positive $\tilde{m}_{\mu}$-measure such that $\gamma K \cap K=\emptyset$ for all $\gamma \in \Gamma$ with $\gamma \neq e$. (We argue as if $\Gamma$ was acting effectively on $\mathscr{G} X$; when it is not the case, we leave the minor appropriate changes to the reader.) For a set $\mathscr{T} \subset \Gamma$ of representatives of $\Gamma_{0} \backslash \Gamma$, the set $\bigsqcup_{\tau \in \mathscr{F}} \tau K$ injects into $\Gamma_{0} \backslash \mathscr{G} X$ and therefore

$$
\left(\sum_{\tau \in \mathscr{T}} \chi(\tau)^{2}\right) \tilde{m}_{\mu}(K)=\tilde{m}_{\mu}\left(\bigsqcup_{\tau \in \mathscr{T}} \tau K\right) \leqslant m_{\mathrm{PS}}\left(\Gamma_{0} \backslash \mathscr{G} X\right)<\infty .
$$

Hence, since $\chi \mid \Gamma_{0}=1$, we obtain.

$$
\sum_{\tau \in \Gamma_{0} \backslash \Gamma} \chi(\tau)^{2}<\infty
$$

For every $\gamma \in \Gamma$, we have thus

$$
\left(\sum_{\tau \in \Gamma_{0} \backslash \Gamma} \chi(\tau)^{2}\right) \chi(\gamma)^{2}=\sum_{\sigma \in \Gamma_{0} \backslash \Gamma} \chi(\sigma)^{2}
$$

which shows first that $\chi(\gamma)^{2}=1$ for all $\gamma \in \Gamma$ and second that $\left|\Gamma_{0} \backslash \Gamma\right|<\infty$.

\section{Maximal tori and actions of lattices with noncommensurable stabilizers}

Let $\mathbb{G}$ be a linear algebraic group defined over $\mathbb{R}$, let $\Gamma<\mathbb{G}(\mathbb{R})$ be a discrete subgroup and set

$\mathscr{T}(\Gamma)=\{\mathbb{T} \subset \mathbb{G} \mid \mathbb{T}$ is a maximal $\mathbb{R}$-split torus such that $\mathbb{T}(\mathbb{R}) /(\mathbb{T}(\mathbb{R}) \cap \Gamma)$ is compact $\}$.

\section{PROPOSITION 5.1}

The $\Gamma$-action by conjugation on $\mathscr{T}(\Gamma)$ is N.C.S.

Here and in the sequel, we will use the following simple lemma.

Lemma 5.2. Let $G$ be a linear algebraic group and let $A_{0}, A_{1}$ be two commensurable subgroups of $\mathbb{G}$. Then $\left(\overline{A_{0}}\right)^{0}=\left(\overline{A_{1}}\right)^{0}$.

Proof of Proposition 5.1. We have to show that, given $\mathbb{T}, \mathbb{T}^{\prime} \in \mathscr{T}(\Gamma)$ such that $\mathscr{N}_{G}(\mathbb{T}) \cap \Gamma$ and $\mathscr{N}_{G}\left(\mathbb{T}^{\prime}\right) \cap \Gamma$ are quasiconjugate in $\Gamma$, then $T$ and $\pi^{\prime}$ are $\Gamma$-conjugate.

First we observe that, for $\mathbb{T} \in \mathscr{T}(\Gamma)$, the group $\left(\mathcal{N}_{\mathrm{G}}(\mathbb{T})(\mathbb{R}) \cap \Gamma\right) /(\mathbb{T}(\mathbb{R}) \cap \Gamma)$ is finite. Indeed, since $\mathbb{}(\mathbb{R}) /(\mathbb{T}(\mathbb{R}) \cap \Gamma)$ is compact, the canonical map

$$
\mathscr{N}_{\mathbb{G}}(\mathbb{T})(\mathbb{R}) /(\mathbb{T}(\mathbb{R}) \cap \Gamma) \rightarrow \mathcal{N}_{\mathbb{G}}(\mathbb{T})(\mathbb{R}) / \mathbb{T}(\mathbb{R})
$$

is proper and therefore $\left(\mathcal{N}_{G}(\mathbb{T})(\mathbb{R}) \cap \Gamma\right) /(\mathbb{J}(\mathbb{R}) \cap \Gamma)$ is a discrete subgroup of the compact group $\mathcal{N}_{G}(\mathbb{T})(\mathbb{R}) / \mathbb{T}(\mathbb{R})$.

If now $\mathscr{N}_{G}(\mathbb{T}) \cap \Gamma$ and $\mathscr{N}_{\mathbb{G}}\left(\mathbb{T}^{\prime}\right) \cap \Gamma$ are quasiconjugate in $\Gamma$, there exist $\Delta<\mathbb{T}(\mathbb{R}) \cap \Gamma$ of finite index and $\gamma \in \Gamma$ such that $\gamma \Delta \gamma^{-1}$ is of finite index in $\Gamma \cap \mathbb{V}^{\prime}(\mathbb{R})$. Passing to

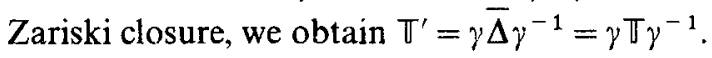


Examples. (1) Let $\mathbb{G}$ be a semisimple $\mathbb{R}$-group and $\Gamma<\mathbb{G}(\mathbb{R})$ a lattice. Then $\mathscr{T}(\Gamma) \neq \emptyset$; this follows from the existence of $\mathbb{R}$-hyper-regular elements in $\Gamma$ [PrR]. Indeed, for such a $\gamma \in \Gamma$, the centralizer $\mathscr{Z}_{G}(\gamma)$ contains an $\mathbb{R}$-split torus $\mathbb{T}$ which is maximal in $\mathbb{G}$ and such that $\mathbb{T}(\mathbb{R}) /(\Gamma \cap \mathbb{T}(\mathbb{R}))$ is compact.

(2) Let $\mathscr{P}$ be the set of primitive indefinite integral binary forms

$$
Q(X, Y)=a X^{2}+b X Y+c Y^{2}
$$

with $a>0$. Then the map which to every $Q \in \mathscr{P}$ associates $S O(Q)^{0}$ gives a bijection between $\mathscr{P}$ and the set of $\mathbb{R}$-split tori $\mathbb{T} \subset S L(2)$ for which $S L(2, \mathbb{Z}) \cap \mathbb{T}(\mathbb{R})$ is a lattice in $\mathbb{T}(\mathbb{R})$ :

$$
\mathscr{P} \cong \mathscr{T}(S L(2, \mathbb{Z})) \text {. }
$$

(3) It is a general fact due to Ono [Ono] that, for a $\mathbb{Q}$-torus $\mathbb{T}$ with $X_{\mathbb{Q}}(\mathbb{\mathbb { T }})=1$, the group $\mathbb{T}(\mathbb{R}) / \mathbb{T}(\mathbb{Z})$ is compact. Hence, given a semisimple $\mathbb{Q}$-group $\mathbb{G}$, the set $\mathscr{T}(\mathbb{G}(\mathbb{Z})$ ) contains all $\mathbb{Q}$-torii $\mathbb{T}$ which are maximal $\mathbb{R}$-split and such that $X_{\mathbb{Q}}(\mathbb{T})=1$. As examples of such torii in $S L(n)$, let $\mathbb{K} / \mathbb{Q}$ be a totally real number field or degree $n$, let $\mathbb{H} \doteqdot \operatorname{Res}_{\mathrm{K} / \mathrm{Q}} \mathbb{G} L_{1} \subset \mathbb{G} L_{n}$ and $\mathbb{I} \doteqdot \mathbb{H} \cap S L(n)$. The group $\mathscr{U}_{\mathrm{K}}$ of units of $\mathbb{K}$ is abelian of rank $n-1$ and isomorphic to $\mathbb{H}(\mathbb{Z})$. As $\mathbb{T}(\mathbb{Z})$ is of index at most two in $\mathbb{H}(\mathbb{Z})$, the torus $\mathbb{T}(\mathbb{Z})$ is of rank $n-1$ and hence $\mathbb{T}(\mathbb{R}) / \mathbb{T}(\mathbb{Z})$ is compact.

\section{Algebraic subgroups and actions of arithmetic lattices with noncommensurable stabilizers}

In this section $\mathbb{G}$ denotes a connected linear algebraic $\mathbb{Q}$-group; let

$$
\mathscr{S}_{\mathbb{G}}=\left\{\mathbb{H} \mid \mathbb{H} \text { is a connected } \mathbb{Q} \text {-subgroup of } \mathbb{G} \text {, of finite index in } \mathcal{N}_{\mathbb{G}}\left(\overline{\mathbb{H}(\mathbb{Z})^{0}}\right)\right\} \text {. }
$$

We will show below that if $\mathbb{H}$ is a connected $\mathbb{Q}$-subgroup of $\mathbb{G}$, one always has the inclusion

$$
\mathbb{H}<\mathscr{N}_{\mathbf{G}}\left(\overline{\mathbb{H}(\mathbb{Z})^{0}}\right)
$$

\section{PROPOSITION 6.1}

The action by conjugation of $\mathbb{G}(\mathbb{Z})$ on $\mathscr{S}_{\mathbb{G}}$ is N.C.S. and $\mathscr{S}_{\mathbb{G}}$ contains all parabolic $\mathbb{Q}$-subgroups of $\mathbb{G}$.

Lemma 6.2. Let $\mathbb{H}$ be a $\mathbb{Q}$-subgroup of $\mathbb{G}$.

(1) $\mathscr{N}_{\mathrm{G}}(\mathrm{H})(\mathbb{Q})<\operatorname{Com}_{\mathrm{G}}(\mathrm{H}(\mathbb{Z}))$

(2) $\mathscr{N}_{\mathrm{G}}(\mathbb{H})^{0}<\mathcal{N}_{\mathrm{G}}\left(\overline{\mathbb{H}(\mathbb{Z})^{0}}\right)$.

Proof of Lemma 6.2. Let us first show the implication $(1) \Longrightarrow(2)$. As $\mathscr{N}_{\mathbb{G}}(\mathbb{H})$ is defined over $\mathbb{Q}$, one has

$$
\mathscr{N}_{G}(\mathbb{H})^{0}<\overline{\mathscr{N}_{G}(\mathbb{H})(\mathbb{Q})}
$$

by a theorem of Rosenlicht [Bor, 18.3]. On the other hand Lemma 5.2 implies

$$
\overline{\operatorname{Com}_{G}(\mathbb{H}(\mathbb{Z}))}<\mathcal{N}_{\mathbb{G}}\left(\overline{\mathbb{H}(\mathbb{Z})^{0}}\right)
$$

and hence (1) implies (2). 
In order to prove (1) we may assume that $\mathbb{H}$ is connected. Let $X_{\mathbf{Q}}(\mathbb{H})$ be the set of Q-characters of $\mathrm{H}-\mathrm{D}$ and set

$$
\mathbb{H}_{0} \doteqdot \bigcap_{\chi \in X_{Q}(\mathbf{H})} \operatorname{Ker} \chi
$$

Clearly, $\mathbb{H}_{0}(\mathbb{Z})$ is a subgroup of finite index in $\mathbb{H}(\mathbb{Z})$ and it follows from $[B H C]$ that $\mathbb{H}_{0}(\mathbb{Z})$ is a lattice in $\mathbb{H}_{0}(\mathbb{R})$. Observe also that $\mathscr{N}_{\mathrm{G}}(\mathbb{H})(\mathbb{Q})$ acts on $X_{\mathbb{Q}}(\mathbb{H})$ and hence normalizes $\mathrm{H}_{0}$.

Let $\mathbb{G}<G L(n, \mathbb{C})$ for some $n$, fix $g \in \mathscr{N}_{\mathbb{G}}(\mathbb{H})(\mathbb{Q})$ and choose an integer $m \geq 1$ such that $m g$ and $m g^{-1}$ are in $M_{n}(\mathbb{Z})$. For the subgroup

$$
\Gamma \doteqdot\left\{\gamma \in \mathbb{H}_{0}(\mathbb{Z}) \mid \gamma \equiv \mathrm{id} \bmod m^{2}\right\}
$$

we have $g \Gamma g^{-1} \subset M_{n}(\mathbb{Z})$ and $\operatorname{det}\left(g \Gamma g^{-1}\right) \subset\{1,-1\}$; hence $g \Gamma g^{-1}<\mathbb{H}_{0}(\mathbb{Z})$. Furthermore, $\Gamma$ is of finite index in $\mathbb{H}_{0}(\mathbb{Z})$ and since $\mathbb{H}_{0}(\mathbb{Z})$ is a lattice in $\mathbb{H}_{0}(\mathbb{R})$, the conjugate $g \Gamma g^{-1}$ is of finite index in $\mathbb{H}_{0}(\mathbb{Z})$ as well. Hence

$$
g \in \operatorname{Com}_{\mathbb{G}}\left(\mathbb{H}_{0}(\mathbb{Z})\right)=\operatorname{Com}_{\mathbb{G}}(\mathbb{H}(\mathbb{Z})) .
$$

Proof of Proposition 6.1. For the first assertion, take $\mathbb{H}_{1}, \mathbb{H}_{2} \in \mathscr{S}_{\mathrm{G}}$ such that $\mathscr{N}_{G}\left(\mathbb{H}_{1}\right)(\mathbb{Z})$ and $\mathscr{N}_{G}\left(\mathbb{H}_{2}\right)(\mathbb{Z})$ are commensurable, hence $\mathscr{N}_{\mathrm{G}}\left(\mathrm{H}_{1}\right)^{0}(\mathbb{Z})$ and $\mathscr{N}_{\mathrm{G}}\left(\mathrm{H}_{2}\right)^{0}(\mathbb{Z})$ are also commensurable. Since $\mathbb{H}_{i}$ is connected, we have $\mathbb{H}_{i}<\mathscr{N}_{G}\left(\mathbb{H}_{i}\right)^{0}$ and since $\mathbb{H}_{i} \in \mathscr{S}_{G}$, Lemma 6.2.2 implies that $\mathbb{H}_{i}$ is of finite index in $\mathscr{N}_{\mathrm{G}}\left(\mathrm{H}_{i}\right)^{0}$, in particular $\mathbb{H}_{1}(\mathbb{Z})$

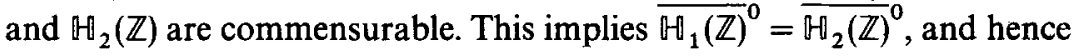

$$
\mathbb{H}_{1}=\mathscr{N}_{G}\left(\left(\overline{\mathbb{H}_{1}(\mathbb{Z})^{0}}\right)^{0}=\mathcal{N}_{\mathrm{G}}\left(\overline{(\mathbb{H}}_{2}(\mathbb{Z})^{0}\right)^{0}=\mathbb{H}_{2}\right. \text {. }
$$

For the second assertion, let $\mathbb{P}$ be a parabolic $\mathbb{Q}$-subgroup of $\mathbb{G}$. Since $\left.\mathbb{P} \subset \mathcal{N}_{\mathrm{G}}(\overline{\mathrm{P}(\mathbb{Z})})^{0}\right)$, the subgroup $\mathbb{P}^{\prime} \doteqdot \mathcal{N}_{\mathbb{G}}\left(\overline{\mathbb{P}(\mathbb{Z})^{0}}\right)$ is $\mathbb{Q}$-parabolic and hence $\mathscr{R}_{u}\left(\mathbb{P}^{\prime}\right) \subset \mathscr{R}_{u}(\mathbb{P})$. Since $\overline{\mathbb{P}(\mathbb{Z})^{0}}$ is normal in $\mathbb{P}^{\prime}$ we have

$$
\mathscr{R}_{u}\left(\overline{\mathbb{P}(\mathbb{Z})}^{0}\right) \subset \mathscr{R}_{u}\left(\mathbb{P}^{\prime}\right)
$$

On the other hand, $\overline{\mathscr{R}_{u}(\mathbb{P})(\mathbb{Z})}=\mathscr{R}_{u}(\mathbb{P})$ and hence $\mathscr{R}_{u}(\mathbb{P})$ is a (normal) subgroup of ${\overline{\mathbb{P}(\mathbb{Z})^{0}}}^{0}$, which implies $\mathscr{R}_{u}\left(\mathbb{P}(\mathbb{Z})^{0}\right) \supset \mathscr{R}_{u}(\mathbb{P})$. This finally shows that $\mathscr{R}_{u}(\mathbb{P})=\mathscr{R}_{u}\left(\mathbb{P}^{\prime}\right)$ and hence $\mathbb{P}=\mathbb{P}^{\prime}$.

Examples. Assume $\mathbb{G}$ to be a semi-simple, defined over $\mathbb{Q}$ and $\mathbb{Q}$-simple. Let $\mathbb{H}$ be a connected semi-simple $\mathbb{Q}$-subgroup of $\mathbb{G}$ which is maximal as a $\mathbb{Q}$-subgroup. Then $\mathbb{H}=\mathscr{N}_{\mathbb{G}}(\mathbb{H})$, and hence $\mathbb{H}=\operatorname{Com}_{\mathrm{G}}(\mathbb{H})$ by Lemma 5.2 . Observe that $\mathbb{G}(\mathbb{Z})$ is a lattice in $\mathbb{G}(\mathbb{R})$ and that $\mathbb{H}(\mathbb{Z})$ is a lattice in $\mathbb{H}(\mathbb{R})$, by $[B H C]$.

Maximal subgroups of the classical groups have been classified by Dynkin [Dyn]. In case $\mathbb{G}$ is $S L(n, \mathbb{C})$ with its standard $\mathbb{Q}$-structure, examples of subgroups $\mathbb{H}$ as above include (to quote but a few):

(i) orthogonal groups $S O(q) \subset S L(n, \mathbb{C})$ for a non degenerate quadratic form $q$ over $\mathbb{Q}$.

(ii) the symplectic group $S p(n, \mathbb{C}) \subset S L(n, \mathbb{C})$ ( $n$ even),

(iii) the images of the fundamental representations $S L(m, \mathbb{C}) \rightarrow S L\left(\left(_{p}^{m}\right), \mathbb{C}\right)$. 


\section{References}

[A'B] A'Campo $\mathbf{N}$ and Burger $\mathbf{M}$, Réseaux arithmétiques et commensurateurs d'après G A Margulis, Invent. Math. 116 (1994) 1-25

[Ada] Adams S, Boundary amenability for word hyperbolic groups and an application to smooth dynamics of simple groups, Topology 33 (1994) 765-783

[BCH] Bekka M, Cowling $M$ and de la Harpe $\mathbf{P}$, Some groups whose reduced $C^{*}$-algebra is simple, Publ. Math. IHES 80 (1994) 117-134

[Bin] Binder M W, On induced representations of discrete groups, Proc. Am. Math. Soc. 118 (1993) 301-309

[Bor] Borel A, Linear algebraic groups, second enlarged edition, Springer, 1991

[BHC] Borel A and Harish-Chandra, Arithmetic subgroups of algebraic groups, Ann. Math. 75 (1962) 485-535

[Bou] Bourdon M, Structure conforme au bord et flot géodésique d'un CAT (-1)-espace, l'Enseignement math. 41 (1995) 63-102

[Bow] Bowditch B H, Geometrical finiteness for hyperbolic groups, J. Funct. Anal. 113 (1993) 245-317

[Boz] Bozejko M, Some aspects of harmonic analysis on free groups. Colloquium Math. 41 (1979) 265-271

[BuM] Burger M and Mozes S, CAT (-1)-spaces, divergence groups and their commensurators. J. Am. Math. Soc. 9 (1996) 57-93

[Coo] Coornaert M, Mesures de Patterson-Sullivan sur le bord d'un espace hyperbolique au sens de Gromov, Pacific J. Math. 159 (1993) 241-270

[CoP] Coornaert $\mathrm{M}$ and Papadopoulos A, Une dichotomie de Hopf pour les flots géodésiques associés aux groupes discrets d'isométrie des arbres, Trans. Am. Math. Soc. 343 (1994) 883-898

[Cor] Corwin L, Induced representation of discrete groups, Proc. Am. Math. Soc. 47 (1975) 279-287

[Dix] Dixmier J, Les C*-algèbres et leurs représentations, Gauthier-Villars, 1969

[Dyn] Dynkin E B, The maximal subgroups of the classical groups (1952), Transl. Am. Math. Soc. Serie 2, 6 (1957) 254-378

[FRZ] Fenn R, Rolfsen D and Zhu J. Centralisers in the braid group and singular braid monoid, Enseignement Math. (to appear)

[Gli] Glimm J, Type I C*-algebras, Ann. Math. 73 (1961) 572-612

[GhH] Ghys E and de la Harpe P, Sur les groupes hyperboliques d'après M Gromov, Birkhäuser, 1990

[Har] de la Harpe P, Groupes hyperboliques, algèbres dopérateurs et un théorème de Jolissaint, C. R. Acad. Sci. Paris, Série I 307 (1988) 771-774

[KrR] Kropholler P H and Roller M A, Relative ends and duality groups, J. Pure Appl. Algebra 61 (1989) 197-210

[Mac] Mackey G W, The theory of unitary group representations, The University of Chicago Press, 1976

[Mar] Margulis G A, Discrete subgroups of semisimple Lie groups, Springer, 1991

[Oba] Obata N, Some remarks on induced representations of infinite discrete groups, Math. Ann. 284 (1989) 91-102

[Ono] Ono T, Sur une propriété arithmétique des groupes commutatifs, Bull. Soc. Math. France 85 (1957) 307-323

[Par] Paris L, Parabolic subgroups of Artin groups, preprint, Dijon (1995)

[Pat] Patterson S J, The limit set of a Fuchsian group, Acta Math. 136 (1976) 241-273

[Ped] Pedersen G K, C*-algebras and their automorphism groups, Academic Press, 1979

[PrR] Prasad G and Raghunathan M S, Cartan subgroups and lattices in semi-simple groups, Ann. Math. 96 (1972) 296-317

[Shi] Shimura G. Introduction to the arithmetic theory of automorphic functions (1971) (Iwanami Shoten and Princeton Univ. Press)

[Sul] Sullivan D, Entropy, Hausdorff measures old and new, and limit sets of geometrically finite Kleinian groups, Acta Math. 153 (1984) 259-277 
[Tho] Thoma E, Ueber unitäre Darstellungen abzählbarer, diskreter Gruppen, Math. Ann. 153 (1964) 111-138

[Yos] Yoshizawa $\mathbf{H}$, Some remarks on unitary representations of the free group, Osaka Math. J. 3 (1951) 55-63 